Nephrol Dial Transplant (2012) 27: 299-303

doi: $10.1093 / \mathrm{ndt} / \mathrm{gfr} 305$

Advance Access publication 30 May 2011

\title{
Event-related distress in kidney disease patients
}

\author{
Sarah Ramer ${ }^{1}$, Anne Germain ${ }^{2}$, Sheena Dohar ${ }^{3}$ and Mark Unruh ${ }^{1}$ \\ ${ }^{1}$ Department of Medicine, Renal-Electrolyte Division, University of Pittsburgh School of Medicine, Pittsburgh, PA, USA, ${ }^{2}$ Department \\ of Psychiatry, University of Pittsburgh School of Medicine, Pittsburgh, PA, USA and ${ }^{3}$ West Virginia University School of Medicine, \\ Morgantown, WV, USA \\ Correspondence and offprint requests to: Sarah Ramer; E-mail ramer.sarah@medstudent.pitt.edu
}

\begin{abstract}
Background. Non-dialysis-dependent chronic kidney disease (CKD) and dialysis-dependent Stage 5 CKD (CKD5) are associated with a significant physical and psychosocial burden. Little is known, however, about the impact of stressful life events on CKD and CKD5 patients. This study aimed to determine the prevalence of stressful life events in CKD and CKD5 patients and identify the factors correlated with high levels of event-related distress.

Methods. This cross-sectional study's sample consisted of 181 patients (91 with non-dialysis-dependent CKD Stages 4 and 5, 90 with CKD5) who filled out the Impact of Event Scale (IES), which measures subjective distress related to stressful life events. Other measures included scores from the Medical Outcomes Study Short Form-36, Patient Health Questionnaire-9 (PHQ-9) and Dialysis Symptom Index (DSI). Results. One hundred and three subjects reported stressors on the IES. Almost half the stressors (49.5\%) related to personal health; the rest fell into other categories. There were significant differences between the no stressor, low event-related distress and high event-related distress groups in age $(\mathrm{P}<0.001)$, PHQ-9 score $(\mathrm{P}<0.001)$ and DSI score $(\mathrm{P}=0.002)$. After adjustment, $\mathrm{PHQ}-9$ score was associated with high event-related distress [odds ratio (OR) 1.20, 95\% confidence interval (CI) 1.10-1.32], as was DSI score (OR $1.04,95 \%$ CI $1.02-1.07)$ in a separate model.

Conclusions. Event-related distress is common in CKD and CKD5 patients. High event-related distress is associated with worse depressive symptoms and greater somatic and emotional symptom burden, even with adjustments for age and gender. The renal practitioner may need to address patients' event-related distress in order to provide optimal care.
\end{abstract}

Keywords: chronic kidney disease; depression; distress; end-stage renal disease; quality of life; symptoms

\section{Introduction}

Though chronic kidney disease (CKD) is generally considered a physical illness, many patients find physical illness accompanied by a significant psychosocial burden. Patients with non-dialysis-dependent $\mathrm{CKD}$ and dialysis-dependent Stage 5 CKD (CKD5) are no exception. Several studies suggest that depression is prevalent among CKD and CKD5 patients $[1,2]$. CKD5 patients are more likely to be hospitalized with a psychiatric disorder than those with at least four other chronic illnesses [3]. And both CKD and CKD5 patients experience a decreased health-related quality of life (HRQoL) compared to healthy people [4]. This psychosocial burden is not without consequences; for example, several studies have linked depression to increased mortality risk in CKD5 patients [5-7].

Little attention, however, has been paid to the psychological impact of specific stressful life events, healthrelated or otherwise, on CKD and CKD5 patients. Stressful life events affect everyone, regardless of whether or not illness is present, but in addition to facing the same events as healthy people, kidney disease patients are subject to the stressful life events connected to suffering from a serious progressive illness and to depending on a machine for survival, in the case of CKD5. Posttraumatic stress disorder (PTSD), a psychiatric condition characterized by symptoms of re-experiencing, avoidance and hyperarousal that persist for $>1$ month, can develop in the wake of a stressful life event [8]. An event that is highly significant to an individual but that does not meet the criteria of trauma as defined by the Diagnostic and Statistical Manual of Mental Disorders-IV (DSM-IV) [8], however, may still be followed by PTSD-like symptoms, such as intrusive thoughts and images of the event or avoidance of reminders of it [911]. These distressing symptoms could add to the already significant psychosocial burden borne by CKD and CKD5 patients and complicate disease-related outcomes.

It is for this reason that we undertook the present study in which we first characterized the prevalence and types of stressful life events reported by patients with CKD and CKD5. Second, since understanding the correlates of event-related distress is essential for both identifying patients likely to suffer from it and developing interventions targeting it, we also examined the extent to which certain clinical factors might be associated with a high level of event-related distress. Third, we evaluated the relationship between event-related distress, depression, symptom burden and HRQoL. 


\section{Materials and Methods}

The study sample was composed of patients with CKD Stages 4 and 5 not dependent on dialysis $(n=91)$, patients undergoing peritoneal dialysis $(n=21)$ and patients undergoing thrice-weekly in-center hemodialysis $(n=69)$ in Pennsylvania. Between March 2004 and May 2008, study staff approached patients to enroll in a study of kidney disease, sleep, mood, cognition and HRQoL at the time of their routine CKD or dialysis clinic visits or their initial evaluations at a kidney transplantation clinic. Though the purpose of their clinic visits differed, all patients either had dialysis-dependent Stage $5 \mathrm{CKD}$ or otherwise advanced CKD (average estimated glomerular filtration rate, calculated using the four-variable Modification of Diet in Renal Disease equation, of $18.7 \mathrm{~mL} / \mathrm{min} / 1.73 \mathrm{~m}^{2}$ ) [12]. Exclusion criteria were age $<18$ years or $>90$ years, active malignancy, active infection (e.g., pneumonia), active coronary artery disease (e.g., unstable angina, myocardial infarction) within the last 6 months, advanced cirrhosis, sleep apnea receiving treatment, advanced dementia, active alcohol abuse and refractory psychiatric disease. This study was approved by the University of Pittsburgh Institutional Review Board and conducted in accordance with the Helsinki Declaration of 1975, as revised in 2000. All participants provided written informed consent.

A total of 298 patients consented to study participation. During home visits, study staff gave questionnaires to the participants to complete and mail back. Of the 298 patients enrolled, 111 did not complete the study assessment ( 51 because of loss of interest or inability of research assistants to make contact to set up appointments, 18 because of failing health, 9 because of missed appointments or non-adherence to study instructions, 9 because of death prior to assessment, 8 because of distances too great for research assistants making home visits or household conditions that interfered with completion of in-home assessments, 6 because of transplantation prior to the first assessment in protocols that required pre- and post-transplant assessments, 5 because of decision not to pursue transplantation in protocols that required post-transplant assessments and 5 because of subject discomfort with other study procedures). Of the remaining 187 participants, 6 did not fill out the Impact of Event Scale (IES), from which the outcome measure for this study was derived, leaving a sample size of 181 subjects.

\section{Self-report measures of event-related distress, mood, symptoms and HRQoL}

Participants completed a series of self-administered questionnaires including the IES, Patient Health Questionnaire-9 (PHQ-9), Dialysis Symptom Index (DSI) and Medical Outcomes Study Short Form-36 (SF-36).

The IES is a 15 -item questionnaire used to measure subjective eventrelated distress in the past week related to a specific life event in the past 6 months named by the respondent. Seven questions measure intrusive symptoms (e.g., unwanted thoughts and images, dreams, strong emotions), while the remaining 8 tap avoidance symptoms (e.g., numbing of responsiveness, attempts to forget or not think about the event, avoidance of potential reminders). Combined, the two dimensions provide a total subjective event-related distress score. Each item is scored, 0 , 'not at all', 1, 'rarely', 3, 'sometimes' or 5, 'often', to describe the frequency of symptoms, for a maximum total score of 75 [13]. The internal consistency, stability, content validity, construct validity, convergent validity and clinical validity of the IES have been demonstrated in multiple studies [14].

While there is no standard classification system in place for events reported on the IES, for the purposes of our intended analysis, we classified the events. Two people (S.R. and a research assistant) independently devised their own classifications based on all the events reported and then met to discuss the reasoning behind their classifications. One author (S.R.) then altered the classifications according to decisions made at the meeting, and the two other authors (A.G. and M.U.) reviewed them for consistency and meaningfulness.

In order to make the concept of event-related distress more clinically useful, we broke IES scores into three categories: no event-related distress, for those subjects who did not report a stressful life event; low eventrelated distress, for those who did report a stressful life event but scored $<35$ on the IES and high event-related distress, for those who reported a stressful life event and scored $\geq 35$. We chose this cut-point of 35 because of evidence that it has the greatest positive predictive value and lowest apparent total misclassification error rate for a diagnosis of PTSD when patients with DSM-III-R-criteria PTSD complete the IES [15]. It is important to note, however, that the IES was never intended as a diagnostic tool for PTSD and, therefore, we did not use the IES with any intention of diagnosing PTSD in our study participants.
The PHQ-9 is a nine-item tool that assesses the frequency with which patients have experienced depressive thoughts or feelings over the prior 2 weeks. The severity of depressive disorder is considered moderate for scores ranging from 10 to 14 , moderately severe for scores of 15-19 and severe for scores of 20-27. In patients on hemodialysis, PHQ-9 scores $>9$ are $92 \%$ sensitive and specific for a diagnosis of depressive disorder [16].

The DSI is a 30-item questionnaire that assesses physical and emotional symptom burden over a 1-week period [17]. Patients answer whether or not they have experienced a particular symptom in the past week, and if they respond positive, they rate the symptom on a scale of 0 , 'not at all' a bother, to 4, 'very much' a bother. Past studies have confirmed the testretest reliability and content validity of the DSI in patients on hemodialysis [17].

The SF-36 is one of the most widely used HRQoL measures. The Physical Component Summary assesses physical well-being, while the Mental Component Summary reflects psychosocial functioning and psychological distress. In the general population, the mean for each summary scale is 50 points, with a standard deviation of 10 points. Higher scores indicate a better quality of life. The SF-36 has been used extensively in patients with kidney disease and has sound psychometric characteristics in this patient population [18-20].

\section{Statistical analyses}

All continuous variables were described using means, variances, maximums and minimums. Frequencies and percentages were used to describe all categorical variables. We tested statistical significance of the differences between groups using two-sampled $t$-tests or analysis of variance (ANOVA) for continuous variables and chi-square or Fisher's exact tests for categorical variables.

In order to account for differences in age and gender, we then employed logistic regression modeling to determine which patient characteristics correlated with high event-related distress. We initially entered into the model all covariates of $\mathrm{P}<0.2$ in univariate analysis and then re-estimated the model after removal of each covariate with $\mathrm{P}>0.1$. The covariate for education was excluded from regression modeling due to the distribution of this covariate, with $<10 \%$ of patients having less than a 12 th grade education. All analyses were performed using SAS (version 9.1; SAS Institute, Cary, NC).

\section{Results}

The 181 patients who completed the study assessments did not differ significantly from the 117 non-participants in age, gender or race (data not shown). Those with CKD5, however, were less likely to complete than those with CKD $(52.9 \%$ versus $77.1 \%, \mathrm{P}<0.001)$. Of the 181 patients who completed the study assessments, $90(49.4 \%)$ had CKD5, $69(76.7 \%)$ on hemodialysis and $21(23.3 \%)$ on peritoneal dialysis. Ninety-one patients $(50.6 \%)$ had CKD.

Of the 181 patients, 103 reported stressors on the IES. Types of stressors are listed in Table 1. Approximately $50 \%$ of the participants reported their personal health as a source of event-related distress. The remaining participants noted stressors in the categories of family member health, family relationship, death, occupational or financial, multiple stressors and miscellaneous, which included events that were not named but were nonetheless rated, as well as some day-to-day hassles and religious references.

Table 2 shows the demographic, health and questionnaire responses of patients by event-related distress category. Patients with higher event-related distress were more likely to be younger (Figure 1), to have at least a 12th grade education and to not have diabetes mellitus. Gender, race, marital status, employment status, smoking status, type of kidney disease (CKD versus CKD5) and presence of cardiovascular disease were not associated with higher eventrelated distress. 
The unadjusted relationships of event-related distress with depressive symptoms and symptom burden are shown in Figures 2 and 3. Patients with higher event-related distress were more likely to have more depressive symptoms, as reported on the PHQ-9 (P<0.001) (Figure 2). However, they were not more likely to have worse HRQoL as measured by the SF-36, either in the Physical or Mental Component Summary, although the latter trended toward significance $(\mathrm{P}=0.06)$. In addition to depressive symptoms, the higher event-related distress patients were also more likely to experience a higher overall symptom burden as reported on the DSI $(\mathrm{P}=0.002)$ (Figure 3$)$.

In order to see if event-related distress was associated with somatic symptoms, we excluded seven psychological and emotional symptoms, the DSI measures ('feeling tired or lack of energy', 'worrying', 'feeling nervous', 'trouble staying asleep', 'feeling irritable', 'feeling sad' and 'feeling anxious'), and re-examined the relationship between eventrelated distress and somatic symptoms. Higher event-

Table 1. Types of stressors reported on the IES ${ }^{\mathrm{a}}$

\begin{tabular}{lc}
\hline Category & $n(\%)$ \\
\hline Personal health & $51(49.5)$ \\
Family relationship & $12(11.7)$ \\
Occupational/financial & $10(9.7)$ \\
Multiple stressors & $10(9.7)$ \\
Miscellaneous/unknown & $9(8.7)$ \\
Family member health & $8(7.8)$ \\
Death & $3(2.9)$ \\
\hline
\end{tabular}

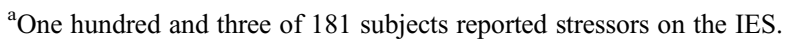

related distress was still associated with a higher overall symptom burden $(n=176, \mathrm{P}=0.02$ in ANOVA, $\mathrm{P}<0.05$ for no stressor versus high event-related distress and low event-related distress versus high event-related distress).

The strength of the associations of event-related distress with mood and symptoms after accounting for age and gender is shown in Table 3 . In a logistic regression model with age and gender as the only covariates entered, neither was associated with a subject's high event-related distress. Depression, however, as measured by the PHQ-9 score, was associated with high event-related distress after adjusting for age and gender [odds ratio (OR) 1.20, 95\% confidence interval (CI) 1.10-1.32]. With adjustment for age and gender in a separate model, dialysis symptoms from the DSI were also associated with high event-related distress (OR 1.04 , 95\% CI 1.02-1.07).

\section{Discussion}

In this study, we found that severe clinically meaningful event-related distress, present in 1/5 of our sample of CKD and CKD5 patients, was associated with worse depressive symptoms and a greater emotional and somatic symptom burden. We further found that event-related distress secondary to many kinds of events is prevalent in CKD and CKD5 patients, with over half of our subjects reporting having experienced a stressful life event in the past 6 months. While roughly half these events related to the patient's own health, the other half was split among several categories of events that people without a serious illness also may face.

Table 2. Characteristics of subjects by event-related distress level ${ }^{\mathrm{a}}$

\begin{tabular}{|c|c|c|c|c|c|}
\hline Category & $\begin{array}{l}\text { Overall } \\
(n=181)\end{array}$ & $\begin{array}{l}\text { No stressor } \\
(n=80)\end{array}$ & $\begin{array}{l}\text { Low event-related distress } \\
(n=65)\end{array}$ & $\begin{array}{l}\text { High event-related distress } \\
(n=36)\end{array}$ & $\mathrm{P}$ \\
\hline Age (years; $n=181$ ) & $52.6 \pm 14.2$ & $57.3 \pm 13.9$ & $49.2 \pm 13.6$ & $48.2 \pm 16.0$ & $<0.001^{\mathrm{b}, \mathrm{c}}$ \\
\hline Male $(\% ; n=181)$ & 61.9 & 71.3 & 55.4 & 52.8 & 0.07 \\
\hline White $(\% ; n=181)$ & 65.8 & 67.5 & 64.6 & 63.9 & 0.9 \\
\hline Married $(\% ; n=179)$ & 59.2 & 62.8 & 55.4 & 58.3 & 0.7 \\
\hline $\begin{array}{l}12 \text { th grade/high school } \\
\text { graduation or more }(\% ; n=179)\end{array}$ & 91.6 & 85.9 & 95.4 & 97.2 & 0.07 \\
\hline $\begin{array}{l}\text { Employed full-time/part-time; } \\
\text { student }(\% ; n=177)\end{array}$ & 32.2 & 24.4 & 39.7 & 36.1 & 0.1 \\
\hline Currently smoking $(\% ; n=181)$ & 13.8 & 15.0 & 9.2 & 19.4 & 0.3 \\
\hline Kidney disease & & & & & 0.4 \\
\hline $\begin{array}{l}\text { CKD Stages } 4 \text { and } 5, \text { not } \\
\text { dialysis dependent }(\% ; n=91)\end{array}$ & 50.3 & 50.0 & 55.4 & 41.7 & \\
\hline $\begin{array}{l}\text { CKD Stage } 5 \text {, dialysis } \\
\text { dependent }(\% ; n=90)\end{array}$ & 49.7 & 50.0 & 44.6 & 58.3 & \\
\hline eGFR $\left(\mathrm{mL} / \mathrm{min} / 1.73 \mathrm{~m}^{2} ; n=85\right)$ & $18.7 \pm 8.0$ & $20.4 \pm 8.4$ & $17.3 \pm 7.3$ & $16.9 \pm 8.1$ & 0.2 \\
\hline \multicolumn{6}{|l|}{ Medical comorbidities } \\
\hline Diabetes mellitus $(\% ; n=181)$ & 37.6 & 46.3 & 35.4 & 22.2 & $0.04^{\mathrm{c}}$ \\
\hline Cardiovascular disease $(\% ; n=179)$ & 31.3 & 39.2 & 26.6 & 22.2 & 0.1 \\
\hline \multicolumn{6}{|l|}{ Short Form-36 score $(n=136)$} \\
\hline Physical Component Summary & $40.7 \pm 6.6$ & $41.1 \pm 6.7$ & $40.8 \pm 6.0$ & $39.6 \pm 7.6$ & 0.6 \\
\hline Mental Component Summary & $44.3 \pm 7.5$ & $44.3 \pm 7.6$ & $45.8 \pm 6.4$ & $41.5 \pm 8.8$ & 0.06 \\
\hline
\end{tabular}

${ }^{a}$ Continuous variables expressed as mean $\pm \mathrm{SD}$. Continuous variables were tested with ANOVA; categorical variables were tested with chi-square or Fisher's exact tests, where appropriate. eGFR, estimated glomerular filtration rate.

${ }^{\mathrm{b}}$ No stressor versus low event-related distress $\mathrm{P}<0.05$ in pairwise comparison.

${ }^{\mathrm{c}}$ No stressor versus high event-related distress $\mathrm{P}<0.05$ in pairwise comparison. 


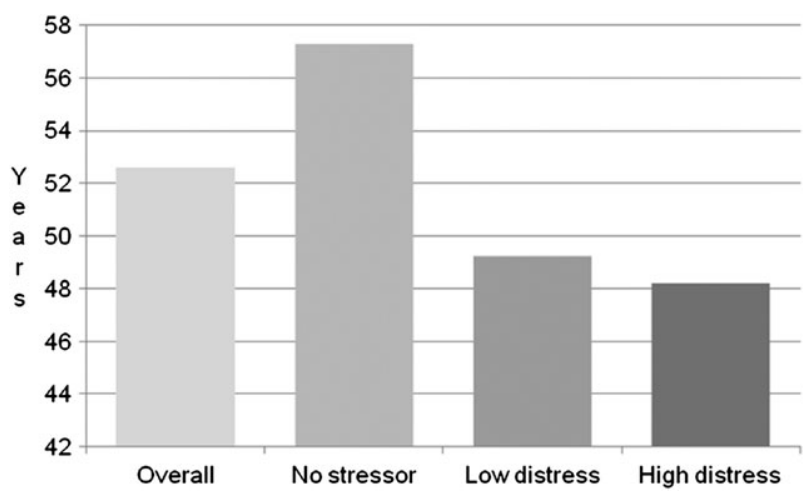

Fig. 1. Mean age by event-related distress level $n=181 ; \mathrm{P}<0.001$ in ANOVA; $\mathrm{P}<0.05$ for no stressor versus low event-related distress and no stressor versus high event-related distress.

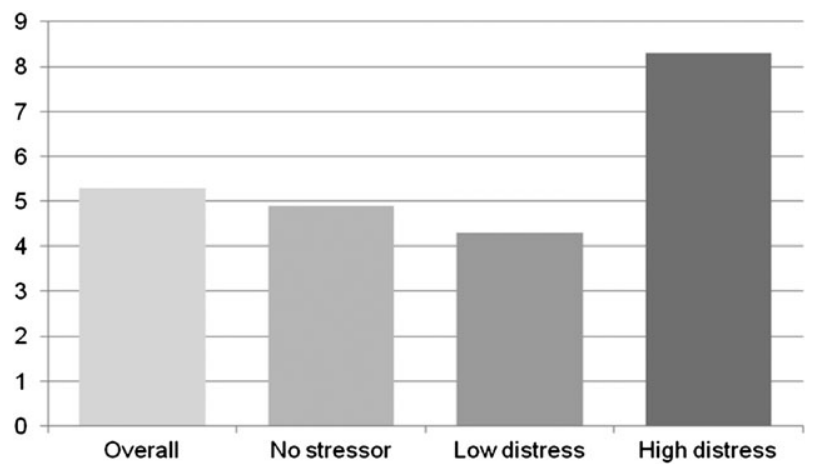

Fig. 2. Mean PHQ-9 score by event-related distress level $n=177$; $\mathrm{P}<$ 0.001 in ANOVA; $\mathrm{P}<0.05$ for no stressor versus high event-related distress and low event-related distress versus high event-related distress.

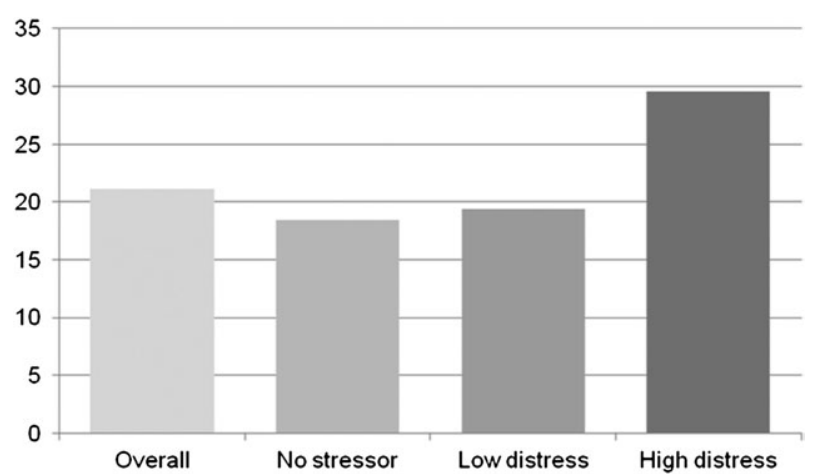

Fig. 3. Mean DSI score by event-related distress level $n=176 ; \mathrm{P}=0.002$ in ANOVA; $\mathrm{P}<0.05$ for no stressor versus high event-related distress and low event-related distress versus high event-related distress.

This report extends our understanding of event-related distress in kidney disease by including CKD and peritoneal dialysis patients from a wide age range (19-85 years old) and diverse racial backgrounds (32\% African-American) and by broadly addressing event-related distress rather than the more narrow category of PTSD. Three previous studies have described event-related distress in kidney disease patients but only in the hemodialysis or post-transplant populations and only event-related distress that met diagnostic criteria for PTSD. Of these studies, the first evaluated the prevalence and predictors of PTSD following Hurricane Katrina among Louisiana residents on hemodialysis [21]. Of the 391 patients who responded to a survey, 24\% reported symptoms consistent with PTSD on the PTSD Checklist [22]. This study, however, only examined PTSD connected to one specific event experienced by all the subjects (i.e., a hurricane). The second study assessed the prevalence of PTSD among 144 German hemodialysis patients using the Posttraumatic Stress Diagnostic Scale and the Impact of Event Scale-Revised [23-25]. This study found that $78 \%$ of subjects had at one time experienced a traumatic life event, lifetime prevalence of PTSD among subjects was $17 \%$ and subjects with PTSD had worse mental health than subjects without. Though this study explored PTSD related to any event, not just one specific event, it also was limited to patients on hemodialysis. The third study examined the effects of PTSD on quality of life among 63 recent kidney transplant recipients from an urban medical center in the USA [26]. Of these patients, $21 \%$ reported symptoms consistent with a PTSD diagnosis and an additional 19\% reported sub-threshold PTSD symptoms. PTSD severity was independently associated with the overall quality of life. Though this study also looked at PTSD related to many types of events, it included only kidney transplant recipients, who for several reasons are a unique population among kidney disease patients. Our work confirms the findings of these earlier studies and then builds on them by showing that event-related distress, at levels comparable to those observed in patients with PTSD, can be detected in patients with both CKD and CKD5.

Our findings should be interpreted in light of certain limitations. We employed a cross-sectional design that does not permit interpretations of causality; longitudinal studies would be necessary to assess the contribution of event-related distress to renal disease outcomes. In addition, this study looked at a sample drawn from a single geographic area, so our findings may not hold for the larger population. Furthermore, although a substantial number of patients in this study suffered from a level of event-related distress that might indicate the presence of PTSD-like reactions, the IES was not designed to diagnose PTSD. More specific assessments are also required to determine whether the stressful life events reported on the IES would meet diagnostic criteria for traumatic events as defined in the DSM-IV diagnostic criteria for PTSD [8]. Therefore, we cannot reach definitive conclusions about PTSD in CKD and CKD5 patients in this study, only about the prevalence and severity of the event-related distress these patients experience.

This study's findings have important implications. First, kidney disease patients struggle with event-related distress from a variety of sources that the clinician may not acknowledge and address unless he employs targeted screening. Moreover, the relationships observed in this study raise the possibility that addressing event-related distress might aid in alleviating the emotional and somatic symptoms experienced by patients with CKD and CKD5. Clinical trials targeting stress and distress management in other chronic diseases, such as HIV/AIDS, diabetes, cancer and cardiovascular disease, have indeed shown improvements in 
Table 3. Predictors of high event-related distress ${ }^{\mathrm{a}}$

\begin{tabular}{|c|c|c|c|}
\hline & $\begin{array}{l}\text { Demographics } \\
\text { only OR } \\
(95 \% \mathrm{CI})\end{array}$ & $\begin{array}{l}\text { Demographics } \\
\text { and PHQ-9 score } \\
\text { OR }(95 \% \mathrm{CI})\end{array}$ & $\begin{array}{l}\text { Demographics } \\
\text { and DSI score } \\
\text { OR }(95 \% \mathrm{CI})\end{array}$ \\
\hline Age & $0.98(0.95-1.00)$ & $0.99(0.96-1.02)$ & $0.98(0.95-1.00)$ \\
\hline Gender & $0.64(0.30-1.34)$ & $0.62(0.27-1.39)$ & $0.88(0.40-1.95)$ \\
\hline $\begin{array}{l}\text { PHQ-9 } \\
\text { score }\end{array}$ & N/A & $1.20(1.10-1.32)$ & $\mathrm{N} / \mathrm{A}$ \\
\hline DSI score & N/A & N/A & $1.04(1.02-1.07)$ \\
\hline
\end{tabular}

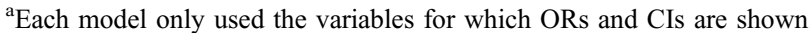
N/A, Not applicable.

disease-related outcomes, mood and functioning [27-33]. Pending evidence from similar trials with CKD and CKD5 patients, renal practitioners seeking to provide optimal care may want to consider the impact of stressful life events on patients, whether or not these events are related to kidney disease.

Acknowledgements. We thank the patients, staff and physicians who supported this study, as well as Irina Karpov for her statistical assistance and Jennifer Alman for her help in classifying events. A portion of this work was previously reported in the abstract "Event-Related Distress in Kidney Disease Patients" at the National Kidney Foundation Spring Clinical Meetings, Nashville, TN, March 26-29, 2009.

Transparency declaration. None of the authors has any relevant financial or other conflicts of interest to declare. The results presented in this paper have not been published previously in whole or part, except in abstract format.

Support and financial disclosure information: S.R. was supported by NIH MH016804, NIH MH019986, Doris Duke Clinical Research Fellowship. A.G. was supported by NIH MH080696. S.D. had no financial support. M.U. was supported by NIH DK066006, NIH DK077785, Fresenius National Kidney Foundation Young Investigator Grant, Paul Teschan Research Grant, Baxter Healthcare. M.U. has consulted for Merck and Baxter Healthcare Medical Advisory Board.

\section{References}

1. Cohen SD, Norris L, Acquaviva K et al. Screening, diagnosis, and treatment of depression in patients with end-stage renal disease. Clin J Am Soc Nephrol 2007; 2: 1332-1342

2. Kimmel PL, Cohen SD, Peterson RA. Depression in patients with chronic renal disease: where are we going? J Ren Nutr 2008; 18: 99-103

3. Kimmel PL, Thamer M, Richard CM et al. Psychiatric illness in patients with end-stage renal disease. Am J Med 1998; 105: 214-221

4. Perlman RL, Finkelstein FO, Liu L et al. Quality of life in chronic kidney disease (CKD): a cross-sectional analysis in the Renal Research Institute-CKD study. Am J Kidney Dis 2005; 45: 658-666

5. Drayer RA, Piraino B, Reynolds CF 3rd et al. Characteristics of depression in hemodialysis patients: symptoms, quality of life and mortality risk. Gen Hosp Psychiatry 2006; 28: 306-312

6. Einwohner R, Bernardini J, Fried L et al. The effect of depressive symptoms on survival in peritoneal dialysis patients. Perit Dial Int 2004; 24: 256-263

7. Kimmel PL, Peterson RA, Weihs KL et al. Multiple measurements of depression predict mortality in a longitudinal study of chronic hemodialysis outpatients. Kidney Int 2000; 57: 2093-2098

8. American Psychiatric Association. Diagnostic and Statistical Manual of Mental Disorders. 4th edn. Washington, DC: American Psychiatric Association; 1994

9. Gold SD, Marx BP, Soler-Baillo JM et al. Is life stress more traumatic than traumatic stress? J Anxiety Disord 2005; 19: 687-698

10. Long ME, Elhai JD, Schweinle A et al. Differences in posttraumatic stress disorder diagnostic rates and symptom severity between Crite- rion A1 and non-Criterion A1 stressors. J Anxiety Disord 2008; 22: 1255-1263

11. Van Hooff M, McFarlane AC, Baur J et al. The stressor Criterion-A1 and PTSD: a matter of opinion? J Anxiety Disord 2009; 23: 77-86

12. Levey AS, Bosch JP, Lewis JB et al. A more accurate method to estimate glomerular filtration rate from serum creatinine: a new prediction equation. Modification of Diet in Renal Disease Study Group. Ann Intern Med 1999; 130: 461-470

13. Horowitz M, Wilner N, Alvarez W. Impact of Event Scale: a measure of subjective stress. Psychosom Med 1979; 41: 209-218

14. Sundin EC, Horowitz MJ. Impact of Event Scale: psychometric properties. Br J Psychiatry 2002; 180: 205-209

15. Neal LA, Busuttil W, Rollins J et al. Convergent validity of measures of post-traumatic stress disorder in a mixed military and civilian population. J Trauma Stress 1994; 7: 447-455

16. Watnick S, Wang PL, Demadura T et al. Validation of 2 depression screening tools in dialysis patients. Am J Kidney Dis 2005; 46: 919-924

17. Weisbord SD, Fried LF, Arnold RM et al. Development of a symptom assessment instrument for chronic hemodialysis patients: the Dialysis Symptom Index. J Pain Symptom Manage 2004; 27: 226-240

18. Edgell ET, Coons SJ, Carter WB et al. A review of health-related quality-of-life measures used in end-stage renal disease. Clin Ther 1996; 18: 887-938

19. Kurtin PS, Davies AR, Meyer KB et al. Patient-based health status measures in outpatient dialysis. Early experiences in developing an outcomes assessment program. Med Care 1992; 30 (5 Suppl): MS136-MS149

20. Meyer KB, Espindle DM, DeGiacomo JM et al. Monitoring dialysis patients' health status. Am J Kidney Dis 1994; 24: 267-279

21. Hyre AD, Cohen AJ, Kutner $\mathrm{N}$ et al. Prevalence and predictors of posttraumatic stress disorder among hemodialysis patients following Hurricane Katrina. Am J Kidney Dis 2007; 50: 585-593

22. Weathers FW, Litz BT, Huska JA et al. PTSD Checklist-Civilian Version. Boston, MA: National Center for PTSD, Behavioral Science Division; 1994

23. Foa EB, Cashman L, Jaycox L et al. The validation of a self-report measure of posttraumatic stress disorder: the Posttraumatic Diagnostic Scale. Psychol Assess 1997; 9: 445-451

24. Maercker A, Schutzwohl M. Erfassung von psychischen Belastungsfolgen: Die Impact of Event Skala-revidierte Version (IES-R). Diagnostica 1998; 44: 130-141

25. Tagay S, Kribben A, Hohenstein A et al. Posttraumatic stress disorder in hemodialysis patients. Am J Kidney Dis 2007; 50: 594-601

26. Possemato K, Geller P, Ouimette P. Posttraumatic stress and quality of life in kidney transplantation recipients. Traumatology 2009; 15: 34-39

27. Amsberg S, Anderbro T, Wredling R et al. A cognitive behavior therapy-based intervention among poorly controlled adult type 1 diabetes patients-A randomized controlled trial. Patient Educ Couns 2009; 77: 72-80

28. Andersson J, Boman K, Jansson JH et al. Effect of intensive lifestyle intervention on C-reactive protein in subjects with impaired glucose tolerance and obesity. Results from a randomized controlled trial with 5-year follow-up. Biomarkers 2008; 13: 671-679

29. Antoni MH, Carrico AW, Duran RE et al. Randomized clinical trial of cognitive behavioral stress management on human immunodeficiency virus viral load in gay men treated with highly active antiretroviral therapy. Psychosom Med 2006; 68: 143-151

30. Antoni MH, Lechner SC, Kazi A et al. How stress management improves quality of life after treatment for breast cancer. J Consult Clin Psychol 2006; 74: 1143-1152

31. Antoni MH, Pereira DB, Marion I et al. Stress management effects on perceived stress and cervical neoplasia in low-income HIV-infected women. J Psychosom Res 2008; 65: 389-401

32. Dusek JA, Hibberd PL, Buczynski B et al. Stress management versus lifestyle modification on systolic hypertension and medication elimination: a randomized trial. J Altern Complement Med 2008; 14: 129-138

33. Fekete EM, Antoni MH, Schneiderman N. Psychosocial and behavioral interventions for chronic medical conditions. Curr Opin Psychiatry 2007; 20: 152-157

Received for publication: 4.11.10; Accepted in revised form: 29.4.11 